\title{
Microtubules as Major Regulators of Endothelial Function: Implication for Lung Injury
}

\author{
Pratap Karki* and Anna A. Birukova
}

Division of Pulmonary and Critical Care Medicine, Department of Medicine, University of Maryland School of Medicine, Baltimore, MD, United States

Endothelial dysfunction has been attributed as one of the major complications in COVID-19 patients, a global pandemic that has already caused over 4 million deaths worldwide. The dysfunction of endothelial barrier is characterized by an increase in endothelial permeability and inflammatory responses, and has even broader implications in the pathogenesis of acute respiratory syndromes such as ARDS, sepsis and chronic illnesses represented by pulmonary arterial hypertension and interstitial lung disease. The structural integrity of endothelial barrier is maintained by cytoskeleton elements, cell-substrate focal adhesion and adhesive cell junctions. Agonist-mediated changes in endothelial permeability are directly associated with reorganization of actomyosin cytoskeleton leading to cell contraction and opening of intercellular gaps or

OPEN ACCESS

Edited by: Alexander Verin, Medical College of Georgia, United States

Reviewed by: Eliana Hiromi Akamine, University of São Paulo, Brazil Jonathan Steven Alexander, Louisiana State University Health

Shreveport, United States

${ }^{*}$ Correspondence: Pratap Kark

pkarki@som.umaryland.edu

Specialty section:

This article was submitted to Vascular Physiology, a section of the journal Frontiers in Physiology

Received: 13 August 2021 Accepted: 06 October 2021 Published: 28 October 2021

Citation:

Karki P and Birukova AA (2021) Microtubules as Major Regulators of Endothelial Function: Implication

for Lung Injury.

Front. Physiol. 12:758313. doi: 10.3389/fphys.2021.758313 enhancement of cortical actin cytoskeleton associated with strengthening of endothelial barrier. The role of actin cytoskeleton remodeling in endothelial barrier regulation has taken the central stage, but the impact of microtubules in this process remains less explored and under-appreciated. This review will summarize the current knowledge on the crosstalk between microtubules dynamics and actin cytoskeleton remodeling, describe the signaling mechanisms mediating this crosstalk, discuss epigenetic regulation of microtubules stability and its nexus with endothelial barrier maintenance, and overview a role of microtubules in targeted delivery of signaling molecules regulating endothelial permeability and inflammation.

Keywords: microtubules, endothelial barrier, cytoskeletal remodeling, inflammation, acute lung injury, histone deacetylase

\section{INTRODUCTION}

Endothelial barrier formed by a lining of heterogenous mixture of endothelial cells (EC) is a highly selective semi-permeable barrier between the blood and interstitial space that is critical for maintaining tissue and organ homeostasis (Bazzoni and Dejana, 2004; Chistiakov et al., 2015). The disruption of EC barrier in lung endothelium results in influx of fluids, macromolecules, pathogens, and immune cells into the lung air spaces, causing pulmonary edema which is a pathological

Abbreviations: MT, microtubules; EC, endothelial cells; ALI, acute lung injury; ARDS, acute respiratory distress syndrome; AJ, adherens junction; TJ, tight junction; MLC, myosin light chain; MYPT1, myosin phosphatase 1; GEF, guanine nucleotide exchange factor; TNF- $\alpha$, tumor necrosis factor- $\alpha$; TGF- $\beta$, transforming growth factor- $\beta$; LPS, lipopolysaccharide; PP2A, protein phosphatase 2 A; PKA, protein kinase A; MAP4, microtubule-associated protein 4; ANP, atrial natriuretic peptide; HGF, hepatocyte growth factor; CS, cyclic stretch; VILI, ventilator-induced lung injury; APC, adenomatous polyposis coli protein; VCAM-1, vascular cell adhesion molecule-1; ICAM-1, intercellular adhesion molecule-1; HDAC6, histone deacetylase 6; SOCS, suppressor of cytokine signaling. 
hallmark of multiple lung diseases such as acute lung injury (ALI), acute respiratory distress syndrome (ARDS), and sepsis (Maniatis et al., 2008; Maniatis and Orfanos, 2008; Opal and van der Poll, 2015). The endothelial barrier dysfunction in other organs have also been implicated in the pathogenesis of various diseases including brain edema, stroke, cognitive impairment, atherosclerosis, metabolic disorders, and cancers (Rodrigues and Granger, 2015; Park-Windhol and D'Amore, 2016; Profaci et al., 2020). More importantly, a number of studies have reported that endothelial barrier dysfunction is a major pathological feature of current global pandemic coronavirus disease 2019 (COVID-19) (Chang et al., 2020; Gavriilaki et al., 2020; Huertas et al., 2020; Jin et al., 2020; Sims et al., 2021). The endothelial barrier integrity is maintained by cell junction adhesive protein complexes linked to actin cytoskeleton, and increasing evidence suggests that microtubules (MT) are integral component of this interactive unit (Sukriti et al., 2014; Kasa et al., 2015). The breach of endothelial barrier is caused by the breakdown of adherens junction (AJ) and/or tight junction (TJ) protein complexes via site-specific protein phosphorylation events leading to the paracellular gap formation. Another mechanism of EC permeability involves Rho GTPasemediated remodeling of actin cytoskeleton and an increase in actomyosin contractility leading to cell retraction and uncoupling of homotypic interactions between transmembrane adhesive proteins (i.e., cadherins, occludins, claudins, nexins) (WojciakStothard and Ridley, 2002; Bazzoni and Dejana, 2004; Wallez and Huber, 2008; Spindler et al., 2010; Komarova et al., 2017). Recent studies have uncovered an equally important role of MT dynamics in regulating endothelial barrier function (Lee and Gotlieb, 2003; Birukova et al., 2004c; Alieva et al., 2013).

Microtubules are dynamic tubular intracellular structures composed of $\alpha$-, $\beta$-tubulin heterodimers and MT-associated regulatory proteins. MT mediate important cellular functions such as cell division, motility, cell shape changes, intracellular transport, and cellular organization (Nogales, 2000; Goodson and Jonasson, 2018). In addition to these conventional functions of MT, changes in MT dynamics caused by vasoactive or inflammatory agonists and mechanical forces play essential role in control of EC permeability via signaling crosstalk with EC junctions and actin cytoskeleton. This review will discuss presently known mechanisms of endothelial barrier regulation by MT signaling nexus.

\section{Microtubules in Control of Endothelial Permeability: Role and Mechanisms}

Cytoskeleton remodeling driven by a balance between contractile and tethering forces determines the endothelial barrier integrity under both physiological and pathological states (Kasa et al., 2015). Endothelial barrier is formed and maintained by intercellular junctions represented by $\mathrm{TJ}$ and $\mathrm{AJ}$ that are composed of multiprotein complexes (Figure 1). A dynamic interaction of these proteins facilitates their association with actin cytoskeleton and maintains the integrity of endothelial barrier. More recent studies have established an essential role of MT in regulating endothelial barrier function since a number

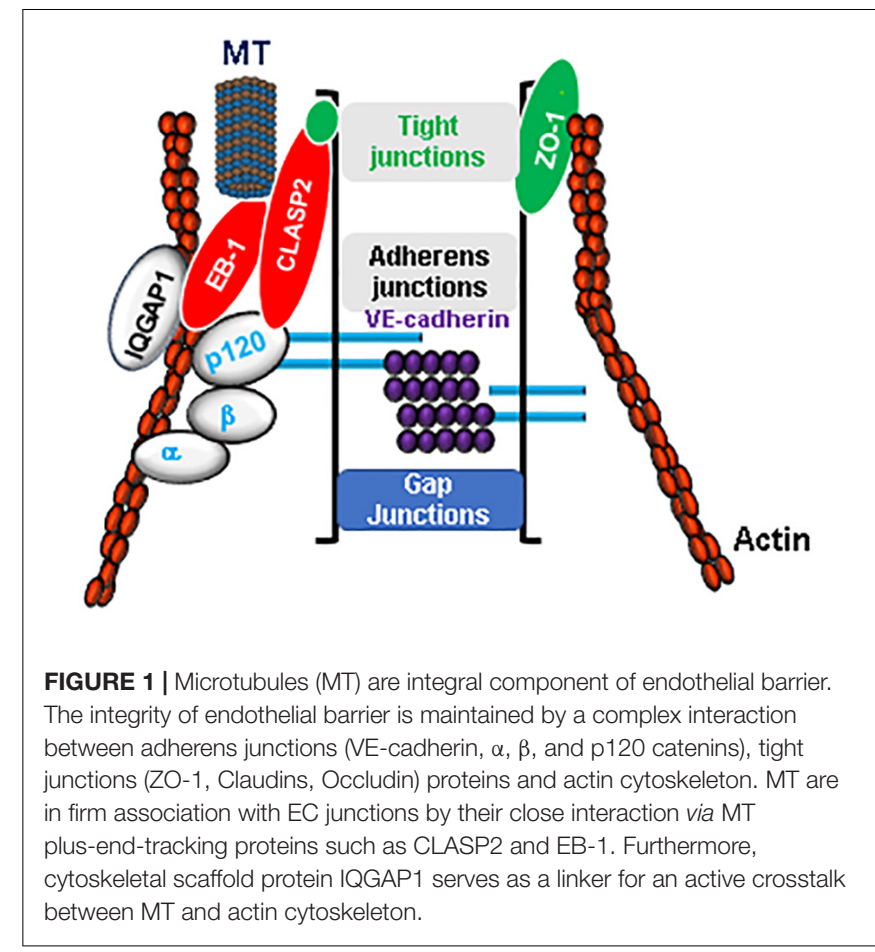

of MT-associated proteins are also involved in dual interaction with EC junction proteins and actin cytoskeleton. As an integral components of cytoskeleton, MT dynamics directly controls endothelial permeability by altering cytoskeletal organization via regulation of actomyosin contractility, paracellular gaps and stress fibers formation (Lee and Gotlieb, 2003; Smurova et al., 2008). More importantly, alteration of MT dynamics appears to be the first target during agonist-induced endothelial barrier disruption. This notion was supported by the findings that thrombin treatment caused peripheral MT disassembly as early as $5 \mathrm{~min}$ but required $30 \mathrm{~min}$ for formation of actin stress fibers (Alieva et al., 2013). These results suggest that changes in MT structure precede microfilaments reorganization during barrier disruptive agents-induced increase in endothelial permeability. Studies have now established that a pool of stable MT favors endothelial barrier integrity, while disassembly of MT network has been associated with an increase in endothelial permeability. In this regard, inhibitors of MT polymerization such as nocodazole directly cause an increase in endothelial permeability which can be prevented by cell pretreatment with MT stabilizer paclitaxel (Verin et al., 2001). In addition to these plant-derived MT poisons, MT growth and MT stability can be also regulated by endogenous circulating bioactive molecules. For example, a rapid and reversible partial dissolution of peripheral MT has been reported in pulmonary EC stimulated with thrombin and is associated with rapid permeability increase (Birukova et al., 2004a). In support of this MT-dependent mechanism, cell pretreatment with MT stabilizing agent taxol rescued thrombininduced increase in endothelial permeability by attenuating Rho activation, suggesting an essential role of MT dynamics 
in controlling endothelial barrier function during agonists challenges. Later on, numerous in vitro and in vivo models of ALI studies, including our own, have provided substantial evidence that MT destabilization is a critical step during endothelial barrier disruption.

Investigations into mechanism of MT-dependent modulation of agonist-induced EC permeability showed that partial depolymerization of peripheral MT promoted, while stabilization prevented activation of the RhoA signaling pathway of endothelial permeability. RhoA GTPase serves as a molecular switch by cycling between GTP-bound active and GDP-bound inactive states and thus regulates various cell functions including actin cytoskeletal reorganization (Siderovski and Willard, 2005; Beckers et al., 2010; Karki and Birukov, 2019). The switch between active and inactive forms of Rho GTPase is regulated by various proteins. Guanine nucleotide exchange factors (GEFs) promote activated, GTP-bound state of RhoA while GDP dissociation inhibitors (GDIs) and GTPase activating proteins (GAPs) inhibit RhoA activity (Siderovski and Willard, 2005). Activated Rho, through its effector Rho kinase, activates actomyosin contractility and EC permeability either directly by phosphorylating myosin light chain (MLC) or indirectly, via inhibition of myosin phosphatase (MYPT1) leading to increased phospho-MLC levels (Garcia et al., 1995; Amano et al., 1996; Kimura et al., 1996). A direct link between Rho activation, MT disassembly and endothelial hyperpermeability was established by the observation that ectopic expression of activated Rho or Rho kinase induced MT disassembly, while overexpression of dominant negative RhoA and Rho-kinase mutants attenuated thrombin-induced peripheral MT dissolution (Birukova et al., 2004a). Consistent with the proposed MT-RhoA-actin cytoskeleton crosstalk mechanism, MT-dependent endothelial barrier disruption was mediated by Rho-mediated MLC phosphorylation, cytoskeletal remodeling, formation of actin stress fibers and paracellular gaps (Verin et al., 2001; Birukova et al., 2004a,c). Further studies revealed that thrombin-induced peripheral MT depolymerization leading to RhoA activation and endothelial permeability was associated with increased MT instability caused by thrombin-induced phosphorylation of MT regulatory protein tau at $\operatorname{Ser}^{409}$ and $\operatorname{Ser}^{262}$ (Birukova et al., 2004a). A similar mechanism of endothelial hyperpermeability was described in pulmonary EC challenged with transforming growth factor-beta1 (TGF- $\beta 1$ ). A partial dissolution of peripheral MT network has also been associated with a decreased pool of acetylated stable MT (Birukova et al., 2005a). Inhibition of the RhoA pathway, MT stabilization or forskolin-mediated elevation of intracellular cAMP attenuated TGF- $\beta 1$-induced MT disassembly and endothelial barrier disruption. Another study demonstrated the protective effects of heat shock protein HSP90 pharmacologic inhibitors against endothelial permeability caused by various agonists including TGF- $\beta 1$ which involved the restoration of peripheral MT organization, inhibition of MLC/MYPT1 phosphorylation and actin stress fibers formation (Antonov et al., 2008).

RhoA-independent mechanism of MT disassembly and subsequent endothelial barrier disruption has also been described. The decrease in a stable pool of MT and a partial dissolution of peripheral MT network during EC permeability caused by tumor necrosis factor- $\alpha$ (TNF- $\alpha$ ) was linked to the activation of p38 mitogen-activated kinase and subsequent destabilization of MT (Petrache et al., 2003). In agreement with these results, inhibition of p38 MAPK directly suppressed nocodazole-induced MT depolymerization and endothelial barrier disruption (Birukova et al., 2005b). Moreover, activation of p38 MAPK mediated 2-Methoxyestradiol-induced MT depolymerization and endothelial barrier disruption (Bogatcheva et al., 2007). A recent report showed that p38 MAPK-dependent phosphorylation of MT-associated protein 4 (MAP4) caused MT disruption, and this mechanism was involved in lipopolysaccharide (LPS) or TNF- $\alpha$-induced endothelial permeability ( $\mathrm{Li}$ et al., 2015). In contrast to RhoA and p38-kinase, activation of protein kinase A (PKA) stabilizes MT network and attenuates nocodazole-induced endothelial barrier dysfunction via suppression of Rho activation, MLC phosphorylation, and stress fiber formation (Birukova et al., 2004b). Similarly, protein phosphatase 2A (PP2A) was found to be associated with MT in endothelium, and inhibition of PP2A caused disassembly of MT via tau phosphorylation leading to an exacerbation of nocodazole-induced endothelial barrier disruption (Tar et al., 2004). Consistently, overexpression of PP2A in ECs protected against thrombin or nocodazoleinduced endothelial barrier disruption via inhibition of tau phosphorylation and MT dissolution (Tar et al., 2006). Exotoxin Y from Pseudomonas aeruginosa is known to increase endothelial permeability (Ochoa et al., 2012). Interestingly, this toxin directly caused MT disassembly and impaired MT reassembly via tau phosphorylation (Balczon et al., 2013).

Oxidative stress-induced alteration of MT dynamics appears to be another common mechanism of endothelial dysfunction caused by bacterial pathogens and inflammatory agonists (Figure 2). For example, LPS-induced increase in reactive oxygen species (ROS) production caused the disassembly of MT, activation of Rho, p38MAPK, and NF- $\kappa$ B signaling pathways leading to endothelial hyperpermeability and inflammation (Kratzer et al., 2012). Likewise, MT destabilization caused by elevated ROS levels has been implicated in the endothelial dysfunction caused by heat-killed Staphylococcus aureus (HKSA) and air pollution particulate matter (Karki et al., 2019a,b). At this point, it is not clear which intermediate molecules that trigger MT destabilization are activated in response to elevated ROS levels. Further studies are also required to explore whether inhibition of ROS production is sufficient for restoration of MT destabilization-induced endothelial dysfunction and if additional mechanisms such as disruption in interaction of MT-associated proteins with other components of EC barrier are involved.

\section{GEF-H1: A Key Player in Microtubules-Mediated Endothelial Dysfunction}

GEF-H1, a Rho-specific GEF, has been identified as a key signaling molecule of an active cross-talk between MT and actin cytoskeleton (Ren et al., 1998; Krendel et al., 2002). GEF-H1 bound to MT remains in an inactive state, but its release 


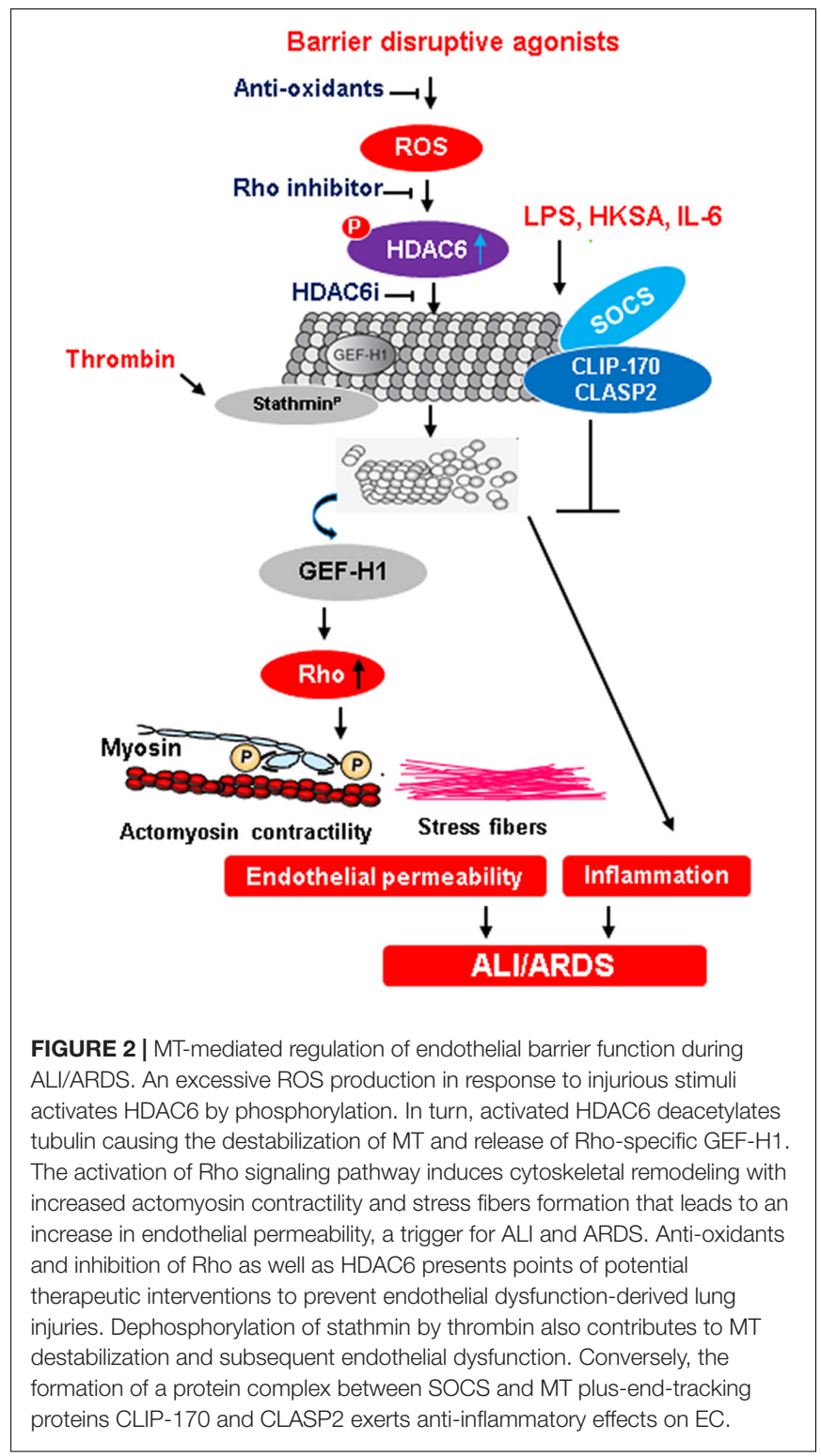

from MT stimulates GEF-H1 nucleotide exchange activity. In consistence with MT-dependent mechanism of GEF-H1 activity regulation, GEF-H1 mutants lacking MT binding sites exhibit increased activity and induce Rho-dependent actin cytoskeleton reorganization (Krendel et al., 2002). A direct role of GEF-H1 in agonist-induced, Rho-dependent and MT disassembly-driven endothelial dysfunction has been clearly elucidated with the findings that knockdown of GEF-H1 attenuated thrombin- or nocodazole-induced EC barrier disruption, while overexpression of wild type GEF-H1 exacerbated such barrier disruptive effects (Birukova et al., 2006). This notion was further validated in cultured human pulmonary EC subjected to high magnitude cyclic stretch $(18 \%$ CS $)$ relevant to ventilatorinduced lung injury (VILI). Exposure to $18 \%$ CS induced MT disassembly and Rho-dependent endothelial dysfunction in pulmonary EC, which was attenuated by knockdown of GEFH1 (Birukova et al., 2010). Protective effects of atrial natriuretic peptide (ANP) against thrombin-induced MT disassembly and Rho-mediated endothelial dysfunction were also mediated by inhibitory phosphorylation of GEF-H1 by Rac1 GTPase effector, PAK1 kinase (Tian et al., 2014a). Thus, a growing body of evidence suggests that activation of GEF-H1 acts as a common mechanism exacerbating pulmonary EC inflammation and barrier dysfunction caused by pathologic mechanical (high magnitude cyclic stretch, increased substrate stiffness) and chemical (LPS, cytokines) stimulation (Mambetsariev et al., 2014; Yi et al., 2017). In addition to MT-associated Rho activator GEF-H1, Rho inhibitor GAP ARHGAP18 localizes on MT and its depletion causes MT destabilization (Lovelace et al., 2017). It was demonstrated that binding of GEF-H1 with TJ protein cingulin rescues thrombin-induced endothelial permeability by inactivating the Rho pathway (Tian et al., 2016). It will be interesting to investigate whether MT dynamics play an essential role in maintaining such interactions to regulate endothelial function.

\section{Microtubules-Associated Rac Signaling in Enhancement of Endothelial Barrier Function}

Basal Rac1 GTPase activity is essential for maintaining endothelial barrier function in resting cells, and its additional activation mediates EC barrier enhancement induced by various barrier protective agents (Mehta and Malik, 2006; Birukova et al., 2007). In this regard, the role of MT-dependent Rac signaling in the regulation of endothelial barrier integrity has been investigated by several groups. The findings from these studies uncovered a crucial role of Asef, a Rac-specific GEF, in MT-dependent endothelial barrier enhancement induced by hepatocyte growth factor (HGF) (Figure 3; Higginbotham et al., 2014; Tian et al., 2014b; Tian X. et al., 2015). Asef has been implicated in cytoskeleton remodeling (Kawasaki et al., 2000, 2010) and it is composed of a Dbl homology domain with GEF activity, plekstrin homology domain determining the subcellular localization, Src homology 3 autoinhibitory domain, and a binding region for tumor suppressor adenomatous polyposis coli protein (APC) (Kawasaki et al., 2003; Gotthardt and Ahmadian, 2007; Murayama et al., 2007). HGF stimulates Rac1-specific activity of Asef by inducing its membrane translocation where it forms a complex with MT-associated protein APC (Tian X. et al., 2015). Moreover, the ectopic expression of constitutively active form of Asef mimics cytoskeletal remodeling induced by HGF and depletion of endogenous Asef or overexpression of dominant negative Asef abolishes the barrier protective effects of HGF, suggesting an essential role of Asef in HGF-induced positive regulation of endothelial barrier (Tian X. et al., 2015). An association of Asef with growing MT tips is essential for HGF-induced endothelial barrier enhancement, as Asef-APC complex is increased in MT enriched fractions upon HGF stimulation (Higginbotham et al., 2014). In turn, inhibition of MT peripheral growth suppresses Asef translocation to cell periphery, resulting in attenuation of Racl activation and subsequent reduction in endothelial barrier enhancement. A critical role of Asef in the regulation of endothelial function 


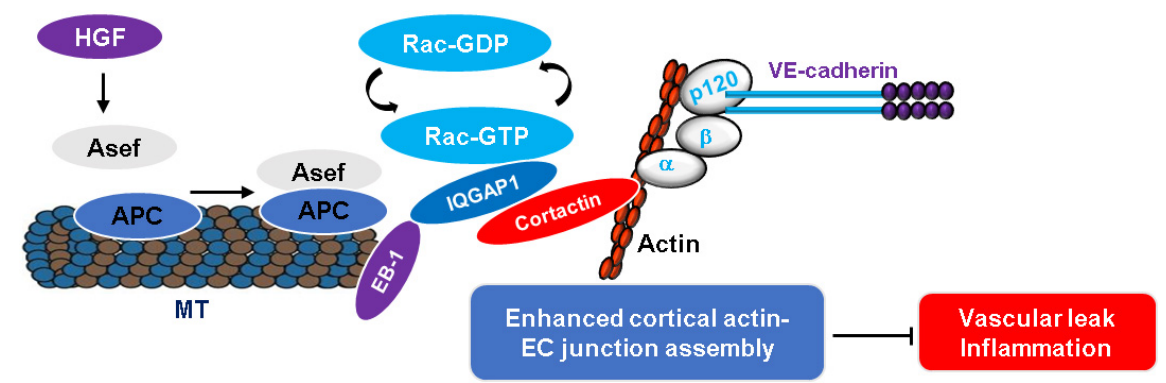

FIGURE 3 | MT-associated Rac regulation of endothelial barrier function. HGF stimulation of EC induces Asef, a Rac-specific GEF, activation which undergoes peripheral translocation by forming a complex with MT protein APC. In turn, Asef associates with IQGAP1 at the cell periphery and activates Rac, resulting in enhanced endothelial barrier integrity. The upregulated endothelial barrier function inhibits agonists-induced vascular leak and inflammation.

was further validated in in vivo studies where HGF-induced protection against LPS- or thrombin-induced lung injury was abolished in Asef knockout mice (Meng et al., 2015; Tian X. et al., 2015).

\section{Microtubules-Associated Proteins in Modulating Endothelial Function}

A number of proteins act as linkers between MT and actin cytoskeleton, thereby regulating MT-mediated cytoskeletal remodeling in response to altered MT dynamics. Among these, a multifunctional adaptor protein IQGAP1 is known to control MT as well as cytoskeletal dynamics by its interaction with Rac as its effector molecule (Swart-Mataraza et al., 2002; Noritake et al., 2005). Furthermore, IQGAP1 also directly interacts with APC and MT plus-end-tracking protein CLIP-170 (Fukata et al., 2002; Watanabe et al., 2004). Interestingly, HGF-induced enhancement of EC barrier function was dependent upon the formation of Asef-Rac-IQGAP1 protein complex at the cell cortical area (Tian Y. et al., 2015). This interaction was facilitated by an interaction between C-terminus of IQGAP1 and active Asef with nucleotide exchange activity. Another MT-associated protein end-binding protein 1 (EB1) also forms a protein complex with IQGAP1 and cortactin following HGF stimulation to mediate the barrier enhancing effects of HGF (Tian et al., 2014c). HGF-stimulated EC showed EB1 distribution to the cell periphery, while EB1 knockdown suppressed Rac1 activation and attenuated HGF-induced enhancement of cortical actin and AJ assembly (Tian et al., 2014c). These findings further strengthened the role of EB1 in coordination of MT-dependent EC barrier function. Stathmin, another MT-associated protein, also controls MT dynamics and agonist-induced endothelial dysfunction. Thrombin-induced dephosphorylation of stathmin disrupts MT network and escalates RhoA-mediated endothelial barrier dysfunction (Tian et al., 2012). Accordingly, knockdown of stathmin attenuated thrombin-induced endothelial permeability in vitro and also suppressed lung vascular leak in the 2-hit mouse model of ALI caused by mechanical ventilation combined with injection of thrombin receptor activating peptide (Tian et al., 2012). The phosphorylation-mediated inactivation of stathmin contributed to ANP-induced restoration of endothelial permeability caused by Staphylococcus aureus-derived peptidoglycan G (Tian Y. I et al., 2015) and was associated with MT destabilization. In that study, ANP suppressed peptidoglycan G-induced MT disassembly, GEF-H1-Rho activation, and inflammatory NF- $\mathrm{B}$ signaling which were abolished upon transient expression of phosphorylation-deficient stathmin mutant. ANP-mediated protection of peptidoglycan G-induced lung injury in mice was associated with increased levels of phosphorylated stathmin, while mice with ANP knockout showed exacerbated lung injury with decreased pools of stable MT and this effect was reversed by stathmin knockdown (Tian Y. I et al., 2015). MAP4 is a cytosolic MT-binding protein that upon phosphorylation disassociates from tubulin and causes MT instability (Ebneth et al., 1999; Hu et al., 2014). As already mentioned above, p38MAPK-mediated serine phosphorylation of MAP4in response to LPS and TNF$\alpha$ stimulation causes MT disruption leading to endothelial permeability (Li et al., 2015).

\section{Microtubules in Endothelial Inflammation}

Excessive inflammatory response is another major feature of endothelial dysfunction that contributes to severe lung injury (Maniatis et al., 2008). Increased endothelial permeability and aggravated inflammation are coordinated responses triggered by pathologic factors and occur in parallel. As such, altered MT dynamics also has been linked to inflammation, and MT stabilizer taxol has been proven to be effective in protecting against both, LPS-induced vascular leak and inflammation in vivo (Mirzapoiazova et al., 2007). Taxol also improved the function of isolated rat lungs during cold preservation that might have significant clinical impacts for lung transplantations (Suzuki et al., 2004). Of note, taxol has been commonly employed as a chemotherapeutic drug against breast, ovarian and lung cancers and its use has been often associated with various side effects including hypersensitivity reactions, neuropathy, myelotoxicity and lung injuries (Raghupathi et al., 2021). In this regard, a study has reported that paclitaxel treated rats show increased inflammatory responses in the lung tissues and an increased vascular permeability with decreased expression of TJ proteins ZO-1 and claudin-4 (Liu et al., 2015). Thus further studies are required to evaluate the beneficial effects of taxol in preventing vascular leak and inflammation during lung injuries. 
The increased expression of EC adhesion molecules intercellular adhesion molecule-1(ICAM-1) and vascular cell adhesion molecule-1 (VCAM-1) is a prerequisite for transendothelial migration of immune cells into affected organs and induce inflammation. It has been reported that ANP-mediated MT stabilization attenuated Peptidoglycan G-induced expression of ICAM-1and VCAM-1 (Tian Y. I et al., 2015). Likewise, other agonists such as HKSA and particulate matter that induce endothelial dysfunction via MT depolymerization are shown to activate inflammatory NF-кB signaling pathway and upregulate the expression of ICAM-1 and VCAM-1 (Karki et al., 2019a,b). A family of suppressor of cytokine signaling (SOCS) proteins also inhibit LPS- and HKSA-induced activation of the NF- $\kappa \mathrm{B}$ pathway and expression of ICAM-1 and VCAM-1 in MT-dependent manner (Karki et al., 2021a,b). These mechanisms will be discussed in the later section. As in the case of permeability, endothelial inflammation may be also mediated by the activation of RhoA pathway, as shown for expression of ICAM-1 induced by thrombin and lysophosphatidic acid (Anwar et al., 2004; Shimada and Rajagopalan, 2010). Nonetheless, the precise role of altered MT dynamics in inducing inflammation is not as clear as in the case of endothelial permeability and future studies are warranted to illustrate the mechanisms of endothelial inflammation promoted by MT disassembly.

\section{Histone Deacetylase 6: Microtubules-Associated Deacetylase as a Therapeutic Target in Lung Injury Syndromes}

Post-translational modifications of tubulin regulates the structure and function of MT (Janke and Bulinski, 2011). In particular, acetylation of tubulin at Lys ${ }^{40}$ confers MT stability (Portran et al., 2017; Xu et al., 2017), whereas HDAC6-mediated deacetylation is linked to destabilization of MT (Hubbert et al., 2002; Matsuyama et al., 2002; Zilberman et al., 2009). HDAC6 is a class IIb HDAC that is exclusively localized in the cytoplasm and directly associates with MT, $\alpha$-tubulin being its preferred substrate (Hubbert et al., 2002; Matsuyama et al., 2002). As therapeutic potential of HDAC6 inhibition has been evaluated in cardiovascular, neurodegenerative diseases and cancers, recent studies have assessed the role of HDAC6 inhibition in endothelial dysfunction-derived ALI (Kovacs et al., 2018; Zhang et al., 2021). Initial studies showed the protective effects of HDAC6 inhibitors against thrombin-induced permeability in EC culture (Saito et al., 2011) and attenuation of LPS-induced ALI in mice (Ni et al., 2010). Likewise, HDAC6 inhibition by its pharmacological inhibitor tubastatin A or siRNA-mediated gene knockdown exerted protection against LPS-induced endothelial barrier disruption and ALI (Joshi et al., 2015). Concurrently, other studies have demonstrated the protective effects of HDA6 inhibition via MT stabilization against other barrier disruptive agents including TNF- $\alpha$ (Yu et al., 2016b) and cigarette smoke extract (Borgas, 2016). These findings are further supported by recent studies where HDAC6 activation-driven MT disruption was a key in mediating endothelial dysfunction caused by Staphylococcus aureus and air pollution particulate matter (Karki et al., 2019a,b). Notably, all aforementioned studies suggest that HDAC6-mediated tubulin deacetylation resulting in MT depolymerization is the major cause of endothelial dysfunction. Briefly, HDAC6-mediated MT destabilization either directly causes the disassembly of AJs or releases GEF-H1 to activate Rho signaling pathway, both ultimately resulting in increased endothelial permeability and inflammation (Borgas, 2016; Yu et al., 2016b; Karki et al., 2019a,b). Elevated ROS production or activated glycogen synthase kinase (GSK)$3 \beta$-mediated serine phosphorylation of HDAC6 appears to act as the upstream activator of HDAC6 (Borgas, 2016; Karki et al., 2019a). Also, a recent study has shown that LPS challenge stimulates HDAC6 activity and HDAC6 inhibitor CAY10603 rescues LPS-induced ALI by restoring tubulin acetylation, and inhibition of cytokine storm and inflammasome (Liu et al., 2019). Nevertheless, a few studies have suggested additional mechanisms of ALI rescue by HDAC6 inhibitors via antiapoptotic and anti-inflammatory activities, reassembly of EC junctions, and inhibition of RhoA signaling (Joshi et al., 2015; Yu et al., 2016a; Liu et al., 2019). A recent study has shown that inhibitors of other HDACs classes also protect against endothelial barrier dysfunction and ALI, but these processes are independent of MT dynamics (Kovacs-Kasa et al., 2021). It indicates that HDAC-mediated epigenetic programming also involves other beneficial mechanisms to combat against endothelial dysfunction beyond HDAC6-mediated control of MT dynamics that needs future attention.

As discussed above, restoration of endothelial functions by barrier-protective agonists involves MT stabilization. In this regard, a recent study has shown that attenuation of LPS-induced endothelial dysfunction caused by unfractionated heparin was associated with restoration of tubulin acetylation levels (Mu et al., 2018). Another study has reported that dopamine D1 receptor (DRD1)-mediated protection of endothelial barrier dysfunction caused by CS involves the inactivation of HDAC6 and maintenance of MT stability (Wang et al., 2021). HDAC6 inhibitors have been also found to be effective against other lung diseases such as pulmonary fibrosis (Saito et al., 2017), and pulmonary arterial hypertension (Boucherat et al., 2017), the conditions associated with altered mechanochemical microenvironment of lung endothelium. Of note, MT stabilization-mediated protection of endothelial barrier integrity is not restricted to lung endothelium as exemplified by taxolinduced protection of corneal EC barrier (Jalimarada et al., 2009; Shivanna and Srinivas, 2009).

Activation of Rho GTPases and HDAC6 appear to act in coordinated manner to cause MT-driven endothelial dysfunction. Activation of RhoA causes MT destabilization via stimulation of HDAC6 activity and conversely HDAC6-driven MT disassembly leads to increased Rho activity, ultimately causing cytoskeletal reorganization associated with endothelial barrier disruption (Schofield et al., 2012). Some proteins are also involved in cross-talk between Rho GTPases and HDAC6 to control MT dynamics. For instance, tubulin polymerization promoting protein 1 (TPP1) favors MT stability by inhibiting HDAC6 activity (Schofield et al., 2013). In turn, Rho-associated coiled-coil 
kinase (ROCK)- mediated phosphorylation of TPP1 abolishes its HDAC6 inhibition activity and inhibits MT polymerization (Schofield et al., 2013).

\section{Suppressor of Cytokine Signaling-Microtubules Interaction: A Novel Microtubules-Mediated Regulatory Mechanism of Endothelial Function}

SOCS represent a family of cytokine-inducible intracellular proteins that act as key regulators of cytokine signaling involved in immunity and inflammation (Alexander, 2002; Yoshimura et al., 2007). The latest studies have unraveled unique mechanisms of SOCS-mediated regulation of endothelial function in MT-dependent fashion (Karki et al., 2021a,b). These studies explored the barrier protective and anti-inflammatory modalities of two closely related SOCS members: SOCS1 and SOCS3 on lung endothelium. The results demonstrated that both SOCS1 and SOCS3 protected against inflammatory agonistinduced endothelial permeability and inflammation in MTdependent manner. SOCS were enriched in MT fractions, while stimulation with inflammatory agonists disrupted SOCS-MT association, and even partial MT disassembly with nocodazole abolished beneficial effects of SOCS on lung EC (Karki et al., 2021a,b). A comprehensive mechanistic analysis showed that SOCS1 and SOCS3, via their N-terminal domains, directly interact with MT plus-end-binding proteins CLIP-170 and CLASP2. More importantly, MT depolymerization not only inhibits the MT-SOCS interaction but also abolishes the protective actions of SOCS on lung endothelium. Multifunctional cytoskeletal scaffold protein IQGAP1 facilitates the delivery, anchoring and stability of anti-inflammatory SOCS-MT signaling complex at the submembrane regions. These findings suggest that proper targeting to MT and interaction with MTassociated proteins is essential for beneficial effects of SOCS on lung endothelium. It indicates that in addition to altered MT dynamics, the interaction of MT proteins with barrier protective molecules is also equally crucial for MT-mediated regulation of endothelial function. It not only establishes an unprecedented role of $\mathrm{MT}$ in regulating anti-inflammatory signaling hub but also warrants further investigation into its mechanistic details and existence of such interaction between MT proteins and inflammation resolving targets under real clinical scenario.

\section{REFERENCES}

Alexander, W. S. (2002). Suppressors of cytokine signalling (SOCS) in the immune system. Nat. Rev. Immunol. 2, 410-416. doi: 10.1038/nri818

Alieva, I. B., Zemskov, E. A., Smurova, K. M., Kaverina, I. N., and Verin, A. D. (2013). The leading role of microtubules in endothelial barrier dysfunction: disassembly of peripheral microtubules leaves behind the cytoskeletal reorganization. J. Cell. Biochem. 114, 2258-2272. doi: 10.1002/jcb. 24575

Amano, M., Ito, M., Kimura, K., Fukata, Y., Chihara, K., Nakano, T., et al. (1996). Phosphorylation and activation of myosin by Rho-associated kinase (Rho-kinase). J. Biol. Chem. 271, 20246-20249. doi: 10.1074/jbc.271.34.2 0246

\section{Summary}

An active and intimate cross-talk between MT and actin cytoskeleton is essential for maintaining endothelial barrier integrity. The altered MT dynamics, specifically depolymerization of $\mathrm{MT}$, is a crucial trigger for endothelial dysfunction and studies have now established that MT destabilization contributes to various barrier disrupting agonists-induced ALI. Accordingly, MT stabilizing drugs and epigenetic modifiers such as HDAC6 inhibitors that favor MT polymerization have shown promising therapeutic potential in a wide range of in vitro and in vivo models of lung injuries. Mechanistically, activation of the Rho signaling pathway evoked by GEF-H1 release from depolymerized MT appears to be the major driving force for MT-derived endothelial dysfunction. On the other hand, MT-dependent Rac activation is crucial for agonist-induced upregulation of endothelial barrier function. Additionally, MT-associated proteins such as stathmin, MAP4 and direct interaction of MT-plus-end tracking proteins CLIP170, CLASP2 with endothelial junction proteins also regulate endothelial barrier function. The latest findings that antiinflammatory proteins SOCS protect endothelial function via their interaction with MT opens a new avenue to pursue such novel MT-dependent mechanisms of EC regulation. In summary, exploring the mechanisms that preserve stable pools of MT and maintain their dynamic interaction with actin to protect endothelial barrier function presents an attractive therapeutic approach to combat against endothelial dysfunctionderived lung injuries such as ALI which still holds an alarming situation with 200,000 cases annually and 30-40\% mortality in the United States.

\section{AUTHOR CONTRIBUTIONS}

$\mathrm{PK}$ and $\mathrm{AB}$ designed the review outline. $\mathrm{PK}$ drafted the manuscript. AB edited the manuscript. Both authors contributed to the article and approved the submitted version.

\section{FUNDING}

The research in authors laboratory and publication of this article is supported by National Institute of Health grants R01 HL107920; R01 HL152761 and R01 HL155051 to AB.

Antonov, A., Snead, C., Gorshkov, B., Antonova, G. N., Verin, A. D., and Catravas J. D. (2008). Heat shock protein 90 inhibitors protect and restore pulmonary endothelial barrier function. Am. J. Respir. Cell. Mol. Biol. 39, 551-559. doi: 10.1165/rcmb.2007-0324OC

Anwar, K. N., Fazal, F., Malik, A. B., and Rahman, A. (2004). RhoA/Rho-associated kinase pathway selectively regulates thrombin-induced intercellular adhesion molecule-1 expression in endothelial cells via activation of I kappa B kinase beta and phosphorylation of RelA/p65. J. Immunol. 173, 6965-6972. doi: 10.4049/ jimmunol.173.11.6965

Balczon, R., Prasain, N., Ochoa, C., Prater, J., Zhu, B., Alexeyev, M., et al. (2013). Pseudomonas aeruginosa exotoxin Y-mediated tau hyperphosphorylation impairs microtubule assembly in pulmonary microvascular endothelial cells. PLoS One 8:e74343. doi: 10.1371/journal.pone.0074343 
Bazzoni, G., and Dejana, E. (2004). Endothelial cell-to-cell junctions: molecular organization and role in vascular homeostasis. Physiol. Rev. 84, 869-901. doi: 10.1152/physrev.00035.2003

Beckers, C. M., van Hinsbergh, V. W., and van Nieuw Amerongen, G. P. (2010). Driving Rho GTPase activity in endothelial cells regulates barrier integrity. Thromb. Haemost. 103, 40-55. doi: 10.1160/TH09-06-0403

Birukova, A. A., Adyshev, D., Gorshkov, B., Bokoch, G. M., Birukov, K. G., and Verin, A. D. (2006). GEF-H1 is involved in agonist-induced human pulmonary endothelial barrier dysfunction. Am J. Physiol. Lung Cell Mol. Physiol. 290, L540-L548. doi: 10.1152/ajplung.00259.2005

Birukova, A. A., Birukov, K. G., Adyshev, D., Usatyuk, P., Natarajan, V., Garcia, J. G., et al. (2005a). Involvement of microtubules and Rho pathway in TGFbetal-induced lung vascular barrier dysfunction. J. Cell. Physiol. 204, 934-947. doi: $10.1002 /$ jcp. 20359

Birukova, A. A., Birukov, K. G., Gorshkov, B., Liu, F., Garcia, J. G., and Verin, A. D. (2005b). MAP kinases in lung endothelial permeability induced by microtubule disassembly. Am. J. Physiol. Lung Cell. Mol. Physiol. 289, L75-L84. doi: 10.1152/ ajplung.00447.2004

Birukova, A. A., Smurova, K., Birukov, K. G., Usatyuk, P., Liu, F., Kaibuchi, K., et al. (2004c). Microtubule disassembly induces cytoskeletal remodeling and lung vascular barrier dysfunction: role of Rho-dependent mechanisms. J. Cell. Physiol. 201, 55-70. doi: 10.1002/jcp.20055

Birukova, A. A., Birukov, K. G., Smurova, K., Adyshev, D., Kaibuchi, K., Alieva, I., et al. (2004a). Novel role of microtubules in thrombin-induced endothelial barrier dysfunction. FASEB J. 18, 1879-1890. doi: 10.1096/fj.04-23 $28 \mathrm{com}$

Birukova, A. A., Liu, F., Garcia, J. G., and Verin, A. D. (2004b). Protein kinase A attenuates endothelial cell barrier dysfunction induced by microtubule disassembly. Am. J. Physiol. Lung Cell. Mol. Physiol. 287, L86-L93. doi: 10.1152/ ajplung.00441.2003

Birukova, A. A., Chatchavalvanich, S., Oskolkova, O., Bochkov, V. N., and Birukov, K. G. (2007). Signaling pathways involved in OxPAPC-induced pulmonary endothelial barrier protection. Microvasc. Res. 73, 173-181. doi: 10.1016/j.mvr. 2006.12.004

Birukova, A. A., Fu, P., Xing, J., Yakubov, B., Cokic, I., and Birukov, K. G. (2010). Mechanotransduction by GEF-H1 as a novel mechanism of ventilator-induced vascular endothelial permeability. Am. J. Physiol. Lung Cell Mol. Physiol. 298, L837-L848. doi: 10.1152/ajplung.00263.2009

Bogatcheva, N. V., Adyshev, D., Mambetsariev, B., Moldobaeva, N., and Verin, A. D. (2007). Involvement of microtubules, p38, and Rho kinases pathway in 2-methoxyestradiol-induced lung vascular barrier dysfunction. Am. J. Physiol. Lung Cell Mol. Physiol. 292, L487-L499. doi: 10.1152/ajplung.0044 1.2003

Borgas, D. (2016). E. Chambers, J. Newton, J. Ko, S. Rivera, S. Rounds, and Q. Lu, Cigarette Smoke Disrupted Lung Endothelial Barrier Integrity and Increased Susceptibility to Acute Lung Injury via Histone Deacetylase 6. Am. J. Respir. Cell. Mol. Biol. 54, 683-696.

Boucherat, O., Chabot, S., Paulin, R., Trinh, I., Bourgeois, A., Potus, F., et al. (2017). HDAC6: a Novel Histone Deacetylase Implicated in Pulmonary Arterial Hypertension. Sci. Rep. 7:4546. doi: 10.1152/ajplung.0021 7.2006

Chang, R., Mamun, A., Dominic, A., and Le, N. T. (2020). SARS-CoV-2 Mediated Endothelial Dysfunction: the Potential Role of Chronic Oxidative Stress. Front. Physiol. 11:605908. doi: 10.1165/rcmb.2015-0149OC

Chistiakov, D. A., Orekhov, A. N., and Bobryshev, Y. V. (2015). Endothelial Barrier and Its Abnormalities in Cardiovascular Disease. Front. Physiol. 6:365. doi: 10.1038/s41598-017-04874-4

Ebneth, A., Drewes, G., Mandelkow, E. M., and Mandelkow, E. (1999). Phosphorylation of MAP2c and MAP4 by MARK kinases leads to the destabilization of microtubules in cells. Cell. Motil. Cytoskeleton 44, 209-224. doi: $10.3389 /$ fphys.2020.605908

Fukata, M., Watanabe, T., Noritake, J., Nakagawa, M., Yamaga, M., Kuroda, S., et al. (2002). Rac1 and Cdc42 capture microtubules through IQGAP1 and CLIP-170. Cell 109, 873-885. doi: 10.3389/fphys.2015.00365

Garcia, J. G., Davis, H. W., and Patterson, C. E. (1995). Regulation of endothelial cell gap formation and barrier dysfunction: role of myosin light chain phosphorylation. J. Cell. Physiol. 163, 510-522. doi: 10.1002/(SICI)10970169(199911)44:3<209::AID-CM6>3.0.CO;2-4
Gavriilaki, E., Anyfanti, P., Gavriilaki, M., Lazaridis, A., Douma, S., and Gkaliagkousi, E. (2020). Endothelial Dysfunction in COVID-19: lessons Learned from Coronaviruses. Curr. Hypertens Rep. 22:63. doi: 10.1016/S00928674(02)00800-0

Goodson, H. V., and Jonasson, E. M. (2018). Microtubules and MicrotubuleAssociated Proteins. Cold Spring Harb. Perspect. Biol. 10:a022608. doi: 10.1002/ jcp.1041630311

Gotthardt, K., and Ahmadian, M. R. (2007). Asef is a Cdc42-specific guanine nucleotide exchange factor. Biol. Chem. 388, 67-71. doi: 10.1007/s11906-02001078-6

Higginbotham, K., Tian, Y., Gawlak, G., Moldobaeva, N., Shah, A., and Birukova, A. A. (2014). Hepatocyte growth factor triggers distinct mechanisms of Asef and Tiam1 activation to induce endothelial barrier enhancement. Cell Signal. 26, 2306-2316. doi: 10.1101/cshperspect.a022608

Hu, J., Chu, Z., Han, J., Zhang, Q., Zhang, D., Dang, Y., et al. (2014). Phosphorylation-dependent mitochondrial translocation of MAP4 is an early step in hypoxia-induced apoptosis in cardiomyocytes. Cell Death Dis. 5:e1424. doi: 10.1515/BC.2007.008

Hubbert, C., Guardiola, A., Shao, R., Kawaguchi, Y., Ito, A., Nixon, A., et al. (2002). HDAC6 is a microtubule-associated deacetylase. Nature 417, 455-458. doi: 10.1016/j.cellsig.2014.07.032

Huertas, A., Montani, D., Savale, L., Pichon, J., Tu, L., Parent, F., et al. (2020). Endothelial cell dysfunction: a major player in SARS-CoV-2 infection (COVID19)? Eur. Respir J. 56:2001634. doi: 10.1038/cddis.2014.369

Jalimarada, S. S., Shivanna, M., Kini, V., Mehta, D., and Srinivas, S. P. (2009). Microtubule disassembly breaks down the barrier integrity of corneal endothelium. Exp. Eye Res. 89, 333-343. doi: 10.1038/417455a

Janke, C., and Bulinski, J. C. (2011). Post-translational regulation of the microtubule cytoskeleton: mechanisms and functions. Nat. Rev. Mol. Cell Biol. 12, 773-786. doi: 10.1183/13993003.01634-2020

Jin, Y., Ji, W., Yang, H., Chen, S., Zhang, W., and Duan, G. (2020). Endothelial activation and dysfunction in COVID-19: from basic mechanisms to potential therapeutic approaches. Signal. Transduct. Target Ther. 5:293. doi: 10.1016/j. exer.2009.03.019

Joshi, A. D., Barabutis, N., Birmpas, C., Dimitropoulou, C., Thangjam, G., CherianShaw, M., et al. (2015). Histone deacetylase inhibitors prevent pulmonary endothelial hyperpermeability and acute lung injury by regulating heat shock protein 90 function. Am. J. Physiol. Lung Cell Mol. Physiol. 309, L1410-L1419. doi: $10.1038 / \mathrm{nrm} 3227$

Karki, P., and Birukov, K. G. (2019). Rho and Reactive Oxygen Species at Crossroads of Endothelial Permeability and Inflammation. Antioxid. Redox. Signal. 31, 1009-1022. doi: 10.1038/s41392-020-00454-7

Karki, P., Cha, B., Zhang, C. O., Li, Y., Ke, Y., Promnares, K., et al. (2021a). Microtubule-dependent mechanism of anti-inflammatory effect of SOCS1 in endothelial dysfunction and lung injury. FASEB J. 35:e21388. doi: 10.1152/ ajplung.00180.2015

Karki, P., Ke, Y., Tian, Y., Ohmura, T., Sitikov, A., Sarich, N., et al. (2019a). Staphylococcus aureus-induced endothelial permeability and inflammation are mediated by microtubule destabilization. J. Biol. Chem. 294, 3369-3384. doi: 10.1089/ars.2019.7798

Karki, P., Ke, Y., Zhang, C. O., Li, Y., Tian, Y., Son, S., et al. (2021b). SOCS3microtubule interaction via CLIP-170 and CLASP2 is critical for modulation of endothelial inflammation and lung injury. J. Biol. Chem. 296:100239. doi: 10.1096/fj.202001477RR

Karki, P., Meliton, A., Sitikov, A., Tian, Y., Ohmura, T., and Birukova, A. A. (2019b). Microtubule destabilization caused by particulate matter contributes to lung endothelial barrier dysfunction and inflammation. Cell. Signal. 53, 246-255. doi: 10.1074/jbc.RA118.004030

Kasa, A., Csortos, C., and Verin, A. D. (2015). Cytoskeletal mechanisms regulating vascular endothelial barrier function in response to acute lung injury. Tissue Barriers 3:e974448. doi: 10.1074/jbc.RA120.014232

Kawasaki, Y., Jigami, T., Furukawa, S., Sagara, M., Echizen, K., Shibata, Y., et al. (2010). The adenomatous polyposis coli-associated guanine nucleotide exchange factor Asef is involved in angiogenesis. J. Biol. Chem. 285, 1199-1207. doi: 10.1016/j.cellsig.2018.10.010

Kawasaki, Y., Sato, R., and Akiyama, T. (2003). Mutated APC and Asef are involved in the migration of colorectal tumour cells. Nat. Cell. Biol. 5, 211-215. doi: $10.4161 / 21688370.2014 .974448$ 
Kawasaki, Y., Senda, T., Ishidate, T., Koyama, R., Morishita, T., Iwayama, Y., et al. (2000). Asef, a link between the tumor suppressor APC and G-protein signaling. Science 289, 1194-1197. doi: 10.1074/jbc.M109.040691

Kimura, K., Ito, M., Amano, M., Chihara, K., Fukata, Y., Nakafuku, M., et al. (1996). Regulation of myosin phosphatase by Rho and Rho-associated kinase (Rho-kinase). Science 273, 245-248. doi: 10.1038/ncb937

Komarova, Y. A., Kruse, K., Mehta, D., and Malik, A. B. (2017). Protein Interactions at Endothelial Junctions and Signaling Mechanisms Regulating Endothelial Permeability. Circ. Res. 120, 179-206. doi: 10.1126/science.289.5482.1194

Kovacs, L., Kovacs-Kasa, A., Verin, A. D., Fulton, D., Lucas, R., and Su, Y. (2018). Histone deacetylases in vascular permeability and remodeling associated with acute lung injury. Vessel Plus 2, 10.20517/2574-1209.2018.06. doi: 10.1126/ science.273.5272.245

Kovacs-Kasa, A., Kovacs, L., Cherian-Shaw, M., Patel, V., Meadows, M. L., Fulton, D. J., et al. (2021). Inhibition of Class IIa HDACs improves endothelial barrier function in endotoxin-induced acute lung injury. J. Cell. Physiol. 236, 28932905. doi: 10.1161/CIRCRESAHA.116.306534

Kratzer, E., Tian, Y., Sarich, N., Wu, T., Meliton, A., Leff, A., et al. (2012). Oxidative stress contributes to lung injury and barrier dysfunction via microtubule destabilization. Am. J. Respir. Cell Mol. Biol. 47, 688-697. doi: 10.20517/25741209.2018.06

Krendel, M., Zenke, F. T., and Bokoch, G. M. (2002). Nucleotide exchange factor GEF-H1 mediates cross-talk between microtubules and the actin cytoskeleton. Nat. Cell. Biol. 4, 294-301. doi: 10.1002/jcp.30053

Lee, T. Y., and Gotlieb, A. I. (2003). Microfilaments and microtubules maintain endothelial integrity. Microsc. Res. Tech. 60, 115-127. doi: 10.1165/rcmb.2012$01610 \mathrm{OC}$

Li, L., Hu, J., He, T., Zhang, Q., Yang, X., Lan, X., et al. (2015). P38/MAPK contributes to endothelial barrier dysfunction via MAP4 phosphorylationdependent microtubule disassembly in inflammation-induced acute lung injury. Sci. Rep. 5:8895. doi: 10.1038/ncb773

Liu, L., Zhou, X., Shetty, S., Hou, G., Wang, Q., and Fu, J. (2019). HDAC6 inhibition blocks inflammatory signaling and caspase-1 activation in LPS-induced acute lung injury. Toxicol. Appl. Pharmacol. 370, 178-183. doi: 10.1002/jemt.10250

Liu, W. J., Zhong, Z. J., Cao, L. H., Li, H. T., Zhang, T. H., and Lin, W. Q. (2015). Paclitaxel-induced lung injury and its amelioration by parecoxib sodium. Sci. Rep. 5:12977. doi: 10.1038/srep08895

Lovelace, M. D., Powter, E. E., Coleman, P. R., Zhao, Y., Parker, A., Chang, G. H., et al. (2017). The RhoGAP protein ARHGAP18/SENEX localizes to microtubules and regulates their stability in endothelial cells. Mol. Biol. Cell. 28, 1066-1078. doi: 10.1016/j.taap.2019.03.017

Mambetsariev, I., Tian, Y., Wu, T., Lavoie, T., Solway, J., Birukov, K. G., et al. (2014). Stiffness-activated GEF-H1 expression exacerbates LPS-induced lung inflammation. PLoS One 9:e92670. doi: 10.1038/srep 12977

Maniatis, N. A., and Orfanos, S. E. (2008). The endothelium in acute lung injury/acute respiratory distress syndrome. Curr. Opin. Crit. Care 14, 22-30. doi: 10.1091/mbc.e16-05-0285

Maniatis, N. A., Kotanidou, A., Catravas, J. D., and Orfanos, S. E. (2008). Endothelial pathomechanisms in acute lung injury. Vascul. Pharmacol. 49, 119-133. doi: 10.1371/journal.pone.0092670

Matsuyama, A., Shimazu, T., Sumida, Y., Saito, A., Yoshimatsu, Y., SeigneurinBerny, D., et al. (2002). In vivo destabilization of dynamic microtubules by HDAC6-mediated deacetylation. EMBO J. 21, 6820-6831. doi: 10.1097/MCC. 0b013e3282f269b9

Mehta, D., and Malik, A. B. (2006). Signaling mechanisms regulating endothelial permeability. Physiol. Rev. 86, 279-367. doi: 10.1016/j.vph.2008.06.009

Meng, F., Meliton, A., Moldobaeva, N., Mutlu, G., Kawasaki, Y., Akiyama, T., et al. (2015). Asef mediates HGF protective effects against LPS-induced lung injury and endothelial barrier dysfunction. Am. J. Physiol. Lung Cell Mol. Physiol. 308, L452-L463. doi: 10.1093/emboj/cdf682

Mirzapoiazova, T., Kolosova, I. A., Moreno, L., Sammani, S., Garcia, J. G., and Verin, A. D. (2007). Suppression of endotoxin-induced inflammation by taxol. Eur. Respir. J. 30, 429-435. doi: 10.1152/physrev.00012.2005

Mu, S., Liu, Y., Jiang, J., Ding, R., Li, X., Li, X., et al. (2018). Unfractionated heparin ameliorates pulmonary microvascular endothelial barrier dysfunction via microtubule stabilization in acute lung injury. Respir. Res. 19:220. doi: 10.1152/ajplung.00170.2014
Murayama, K., Shirouzu, M., Kawasaki, Y., Kato-Murayama, M., HanawaSuetsugu, K., Sakamoto, A., et al. (2007). Crystal structure of the rac activator, Asef, reveals its autoinhibitory mechanism. J. Biol. Chem. 282, 4238-4242. doi: 10.1183/09031936.00154206

Ni, Y. F., Wang, J., Yan, X. L., Tian, F., Zhao, J. B., Wang, Y. J., et al. (2010). Histone deacetylase inhibitor, butyrate, attenuates lipopolysaccharide-induced acute lung injury in mice. Respir. Res. 11:33. doi: 10.1186/s12931-018-0925-6

Nogales, E. (2000). Structural insights into microtubule function. Annu. Rev. Biochem. 69, 277-302. doi: 10.1074/jbc.C600234200

Noritake, J., Watanabe, T., Sato, K., Wang, S., and Kaibuchi, K. (2005). IQGAP1: a key regulator of adhesion and migration. J Cell Sci 118, 2085-2092. doi: 10.1186/1465-9921-11-33

Ochoa, C. D., Alexeyev, M., Pastukh, V., Balczon, R., and Stevens, T. (2012). Pseudomonas aeruginosa exotoxin $\mathrm{Y}$ is a promiscuous cyclase that increases endothelial tau phosphorylation and permeability. J. Biol. Chem. 287, 2540725418. doi: 10.1146/annurev.biochem.69.1.277

Opal, S. M., and van der Poll, T. (2015). Endothelial barrier dysfunction in septic shock. J. Intern. Med. 277, 277-293. doi: 10.1242/jcs.02379

Park-Windhol, C., and D'Amore, P. A. (2016). Disorders of Vascular Permeability. Annu. Rev. Pathol. 11, 251-281. doi: 10.1074/jbc.M111.301440

Petrache, I., Birukova, A., Ramirez, S. I., Garcia, J. G., and Verin, A. D. (2003). The role of the microtubules in tumor necrosis factor-alpha-induced endothelial cell permeability. Am. J. Respir. Cell Mol. Biol. 28, 574-581. doi: 10.1111/joim.12331

Portran, D., Schaedel, L., Xu, Z., Thery, M., and Nachury, M. V. (2017). Tubulin acetylation protects long-lived microtubules against mechanical ageing. Nat. Cell. Biol. 19, 391-398. doi: 10.1146/annurev-pathol-012615-044506

Profaci, C. P., Munji, R. N., Pulido, R. S., and Daneman, R. (2020). The bloodbrain barrier in health and disease: important unanswered questions. J. Exp. Med. 217:e20190062. doi: 10.1165/rcmb.2002-0075OC

Raghupathi, R., Patil, C., and Dhiman, P. (2021). Drug-induced infiltrative lung disease with weekly paclitaxel in breast cancer: Case series. J. Cancer Res. Ther. 17, 262-265. doi: 10.1038/ncb3481

Ren, Y., Li, R., Zheng, Y., and Busch, H. (1998). Cloning and characterization of GEF-H1, a microtubule-associated guanine nucleotide exchange factor for Rac and Rho GTPases. J. Biol. Chem. 273, 34954-34960. doi: 10.1084/jem.20190062

Rodrigues, S. F., and Granger, D. N. (2015). Blood cells and endothelial barrier function. Tissue Barriers 3:e978720. doi: 10.4103/jcrt.JCRT_1225_16

Saito, S., Lasky, J. A., Guo, W., Nguyen, H., Mai, A., Danchuk, S., et al. (2011). Pharmacological inhibition of HDAC6 attenuates endothelial barrier dysfunction induced by thrombin. Biochem. Biophys. Res. Commun. 408, 630634. doi: $10.1074 /$ jbc.273.52.34954

Saito, S., Zhuang, Y., Shan, B., Danchuk, S., Luo, F., Korfei, M., et al. (2017). Tubastatin ameliorates pulmonary fibrosis by targeting the TGFbeta-PI3K-Akt pathway. PLoS One 12:e0186615. doi: 10.4161/21688370.2014.978720

Schofield, A. V., Gamell, C., Suryadinata, R., Sarcevic, B., and Bernard, O. (2013). Tubulin polymerization promoting protein 1 (Tppp1) phosphorylation by Rhoassociated coiled-coil kinase (rock) and cyclin-dependent kinase 1 (Cdk1) inhibits microtubule dynamics to increase cell proliferation. J. Biol. Chem. 288, 7907-7917. doi: 10.1016/j.bbrc.2011.04.075

Schofield, A. V., Steel, R., and Bernard, O. (2012). Rho-associated coiled-coil kinase (ROCK) protein controls microtubule dynamics in a novel signaling pathway that regulates cell migration. J. Biol. Chem. 287, 43620-43629. doi: 10.1371/ journal.pone.0186615

Shimada, H., and Rajagopalan, L. E. (2010). Rho kinase-2 activation in human endothelial cells drives lysophosphatidic acid-mediated expression of cell adhesion molecules via NF-kappaB p65. J. Biol. Chem. 285, 12536-12542. doi: 10.1074/jbc.M112.441048

Shivanna, M., and Srinivas, S. P. (2009). Microtubule stabilization opposes the (TNF-alpha)-induced loss in the barrier integrity of corneal endothelium. Exp. Eye Res. 89, 950-959. doi: 10.1074/jbc.M112.394965

Siderovski, D. P., and Willard, F. S. (2005). The GAPs, GEFs, and GDIs of heterotrimeric G-protein alpha subunits. Int. J. Biol. Sci. 1, 51-66. doi: 10.1074/ jbc.M109.099630

Sims, J. T., Krishnan, V., Chang, C. Y., Engle, S. M., Casalini, G., Rodgers, G. H., et al. (2021). Characterization of the cytokine storm reflects hyperinflammatory endothelial dysfunction in COVID-19. J. Allergy Clin. Immunol. 147, 107-111. doi: 10.1016/j.exer.2009.08.004 
Smurova, K. M., Biriukova, A. A., Verin, A. D., and Alieva, I. B. (2008). [The microtubule system in endothelial barrier dysfunction: disassembly of peripheral microtubules and microtubules reorganization in internal cytoplasm]. Tsitologiia 50, 49-55. doi: 10.7150/ijbs.1.51

Spindler, V., Schlegel, N., and Waschke, J. (2010). Role of GTPases in control of microvascular permeability. Cardiovasc. Res. 87, 243-253. doi: 10.1016/j.jaci. 2020.08.031

Sukriti, S., Tauseef, M., Yazbeck, P., and Mehta, D. (2014). Mechanisms regulating endothelial permeability. Pulm. Circ. 4, 535-551. doi: 10.1134/ S1990519X08010070

Suzuki, S., Bing, H., Sugawara, T., Matsuda, Y., Tabata, T., Hoshikawa, Y., et al. (2004). Paclitaxel prevents loss of pulmonary endothelial barrier integrity during cold preservation. Transplantation 78, 524-529. doi: 10.1093/cvr/ cvq086

Swart-Mataraza, J. M., Li, Z., and Sacks, D. B. (2002). IQGAP1 is a component of Cdc42 signaling to the cytoskeleton. J. Biol. Chem. 277, 24753-24763. doi: $10.1086 / 677356$

Tar, K., Birukova, A. A., Csortos, C., Bako, E., Garcia, J. G., and Verin, A. D. (2004). Phosphatase 2A is involved in endothelial cell microtubule remodeling and barrier regulation. J. Cell. Biochem. 92, 534-546. doi: 10.1097/01.TP. 0000131951.72851 .57

Tar, K., Csortos, C., Czikora, I., Olah, G., Ma, S. F., Wadgaonkar, R., et al. (2006). Role of protein phosphatase $2 \mathrm{~A}$ in the regulation of endothelial cell cytoskeleton structure. J. Cell. Biochem. 98, 931-953. doi: 10.1074/jbc.M1111 65200

Tian, X., Tian, Y., Gawlak, G., Meng, F., Kawasaki, Y., Akiyama, T., et al. (2015). Asef controls vascular endothelial permeability and barrier recovery in the lung. Mol. Biol. Cell. 26, 636-650. doi: 10.1002/jcb.20036

Tian, X., Tian, Y., Gawlak, G., Sarich, N., Wu, T., and Birukova, A. A. (2014a). Control of vascular permeability by atrial natriuretic peptide via a GEF-H1dependent mechanism. J. Biol. Chem. 289, 5168-5183. doi: 10.1002/jcb.2 0829

Tian, X., Tian, Y., Moldobaeva, N., Sarich, N., and Birukova, A. A. (2014b). Microtubule dynamics control HGF-induced lung endothelial barrier enhancement. PLoS One 9:e105912. doi: 10.1091/mbc.E14-02-0725

Tian, X., Tian, Y., Sarich, N., Wu, T., and Birukova, A. A. (2012). Novel role of stathmin in microtubule-dependent control of endothelial permeability. FASEB J. 26, 3862-3874. doi: 10.1074/jbc.M113.493924

Tian, Y. I, Mambetsariev, N. Sarich, Meng, F., and Birukova, A. A. (2015). Role of microtubules in attenuation of PepG-induced vascular endothelial dysfunction by atrial natriuretic peptide. Biochim. Biophys. Acta 1852, 104-119. doi: 10 . 1371/journal.pone.0105912

Tian, Y., Gawlak, G., Shah, A. S., Higginbotham, K., Tian, X., Kawasaki, Y., et al. (2015). Hepatocyte growth factor-induced Asef-IQGAP1 complex controls cytoskeletal remodeling and endothelial barrier. J. Biol. Chem. 290, 4097-4109. doi: 10.1096/fj.12-207746

Tian, Y., Gawlak, G., Tian, X., Shah, A. S., Sarich, N., Citi, S., et al. (2016). Role of Cingulin in Agonist-induced Vascular Endothelial Permeability. J. Biol. Chem. 291, 23681-23692. doi: 10.1016/j.bbadis.2014.10.012

Tian, Y., Tian, X., Gawlak, G., O’Donnell, J. J., Sacks, D. B., and Birukova, A. A. (2014c). IQGAP1 regulates endothelial barrier function via EB1-cortactin cross talk. Mol. Cell. Biol. 34, 3546-3558. doi: 10.1074/jbc.M114.620377

Verin, A. D., Birukova, A., Wang, P., Liu, F., Becker, P., Birukov, K., et al. (2001). Microtubule disassembly increases endothelial cell barrier dysfunction: role of MLC phosphorylation. Am. J. Physiol. Lung Cell Mol. Physiol 281, L565-L574. doi: $10.1074 /$ jbc.M116.720763
Wallez, Y., and Huber, P. (2008). Endothelial adherens and tight junctions in vascular homeostasis, inflammation and angiogenesis. Biochim. Biophys. Acta 1778, 794-809. doi: 10.1128/MCB.00248-14

Wang, Y., Liu, Y. J., Xu, D. F., Zhang, H., Xu, C. F., Mao, Y. F., et al. (2021). DRD1 downregulation contributes to mechanical stretch-induced lung endothelial barrier dysfunction. Theranostics 11, 2505-2521. doi: 10.1152/ajplung.2001. 281.3.L565

Watanabe, T., Wang, S., Noritake, J., Sato, K., Fukata, M., Takefuji, M., et al. (2004). Interaction with IQGAP1 links APC to Rac1, Cdc42, and actin filaments during cell polarization and migration. Dev. Cell. 7, 871-883. doi: 10.1016/j.bbamem. 2007.09.003

Wojciak-Stothard, B., and Ridley, A. J. (2002). Rho GTPases and the regulation of endothelial permeability. Vascul. Pharmacol. 39, 187-199. doi: 10.7150/thno. 46192

Xu, Z., Schaedel, L., Portran, D., Aguilar, A., Gaillard, J., Marinkovich, M. P., et al. (2017). Microtubules acquire resistance from mechanical breakage through intralumenal acetylation. Science 356, 328-332. doi: 10.1016/j.devcel.2004. 10.017

Yi, L., Huang, X., Guo, F., Zhou, Z., Chang, M., and Huan, J. (2017). GSK-3BetaDependent Activation of GEF-H1/ROCK Signaling Promotes LPS-Induced Lung Vascular Endothelial Barrier Dysfunction and Acute Lung Injury. Front. Cell Infect. Microbiol. 7:357. doi: 10.1016/S1537-1891(03)00008-9

Yoshimura, A., Naka, T., and Kubo, M. (2007). SOCS proteins, cytokine signalling and immune regulation. Nat. Rev. Immunol. 7, 454-465. doi: 10.1126/science. aai8764

Yu, J., Ma, M., Ma, Z., and Fu, J. (2016a). HDAC6 inhibition prevents TNF-alphainduced caspase 3 activation in lung endothelial cell and maintains cell-cell junctions. Oncotarget 7, 54714-54722. doi: 10.3389/fcimb.2017.00357

Yu, J., Ma, Z., Shetty, S., Ma, M., and Fu, J. (2016b). Selective HDAC6 inhibition prevents TNF-alpha-induced lung endothelial cell barrier disruption and endotoxin-induced pulmonary edema. Am. J. Physiol. Lung Cell. Mol. Physiol. 311, L39-L47. doi: 10.1038/nri2093

Zhang, Q., Wang, Y., Qu, D., Yu, J., and Yang, J. (2021). Role of HDAC6 inhibition in sepsis-induced acute respiratory distress syndrome (Review). Exp. Ther. Med. 21:422. doi: 10.18632/oncotarget.10591

Zilberman, Y., Ballestrem, C., Carramusa, L., Mazitschek, R., Khochbin, S., and Bershadsky, A. (2009). Regulation of microtubule dynamics by inhibition of the tubulin deacetylase HDAC6. J. Cell. Sci. 122, 3531-3541. doi: 10.1152/ajplung. 00051.2016

Conflict of Interest: The authors declare that the research was conducted in the absence of any commercial or financial relationships that could be construed as a potential conflict of interest.

Publisher's Note: All claims expressed in this article are solely those of the authors and do not necessarily represent those of their affiliated organizations, or those of the publisher, the editors and the reviewers. Any product that may be evaluated in this article, or claim that may be made by its manufacturer, is not guaranteed or endorsed by the publisher.

Copyright (c) 2021 Karki and Birukova. This is an open-access article distributed under the terms of the Creative Commons Attribution License (CC BY). The use, distribution or reproduction in other forums is permitted, provided the original author(s) and the copyright owner(s) are credited and that the original publication in this journal is cited, in accordance with accepted academic practice. No use, distribution or reproduction is permitted which does not comply with these terms. 